\title{
GENETIC CONTENT AND BUOYANT DENSITY OF RABBIT SPERMATOZOA
}

\author{
R. A. BEATTY \\ A.R.C. Unit of Animal Genetics, Institute of Animal Genetics, \\ West Mains Road, Edinburgh 9
}

(Received 3rd March 1969)

The possibility of controlling the transmission of heritable characters by differential separation, inactivation or destruction of spermatozoa according to their individual genetic content depends on whether the phenotype of the developing or mature spermatozoon is affected by its own haploid genetic content. The present work shows no evidence of any such 'haploid effect' on the buoyant density of spermatozoa attributable to the segregation of three simple Mendelian factors (sex, albino and rex locus alleles) or to the segregation of factors determining viability of young at birth, and no certain effect among the various genes governing birth weight.

The living spermatozoa of whole single ejaculates were fractionated into different buoyant density classes by centrifugation to near-equilibrium after layering upon 4-ml dextran-based density gradients each covering a range of 0.02 specific gravity units and giving optimum motility. Tester does were inseminated with aliquots of the sedimented fraction (taken up in a saline) and of either the whole (mixed) suspended fraction or else its upper and lower halves (each mixed). The technical details of this same experiment have already been recorded by Beatty (1964a), the present paper being a sequel, and an absence of any effect on the sex ratio in the initial results was reported briefly (Beatty, 1964b); it was also known that spermatozoa fell into distinct bands in density gradients. Some properties of the density gradient medium are shown in Text-fig. 1. The buoyant densities obtained with this medium were thought to be close to the true specific gravities or else a little too high, and are of the same order as those recorded by Lavon, Volcani, Amir \& Danon (1966). Other workers tend to find much higher buoyant densities, perhaps because non-physiological media have frequently been employed, often enormously hypertonic and/or lethal. The present medium was elaborated specifically for the purpose of keeping osmotic pressure and $\mathrm{pH}$ within physiological limits.

Semen was collected from rabbits of genetic constitution $c c^{\text {cht }} R r$ from the Edinburgh-AS stock; i.e. heterozygous for albino and the partial dominant light chinchilla and for non-rex fur and the fully recessive rex. All were homozygous non-agouti, non-brown and non-dilute. Tester does were $c c r r$, except that some had unsuitable constitutions at one or both loci and yielded only partial information. On the day of birth, offspring were weighed, sexed by 
dissection, and their phenotypes scored. Any experimental separation of $\mathrm{X}$ from Y spermatozoa or $c$ from $c^{\text {chl }}$ or $R$ from $r$ would have caused departures from the expected Mendelian ratios. Control natural matings from the males of the experiment were interspersed between the experimental inseminations.

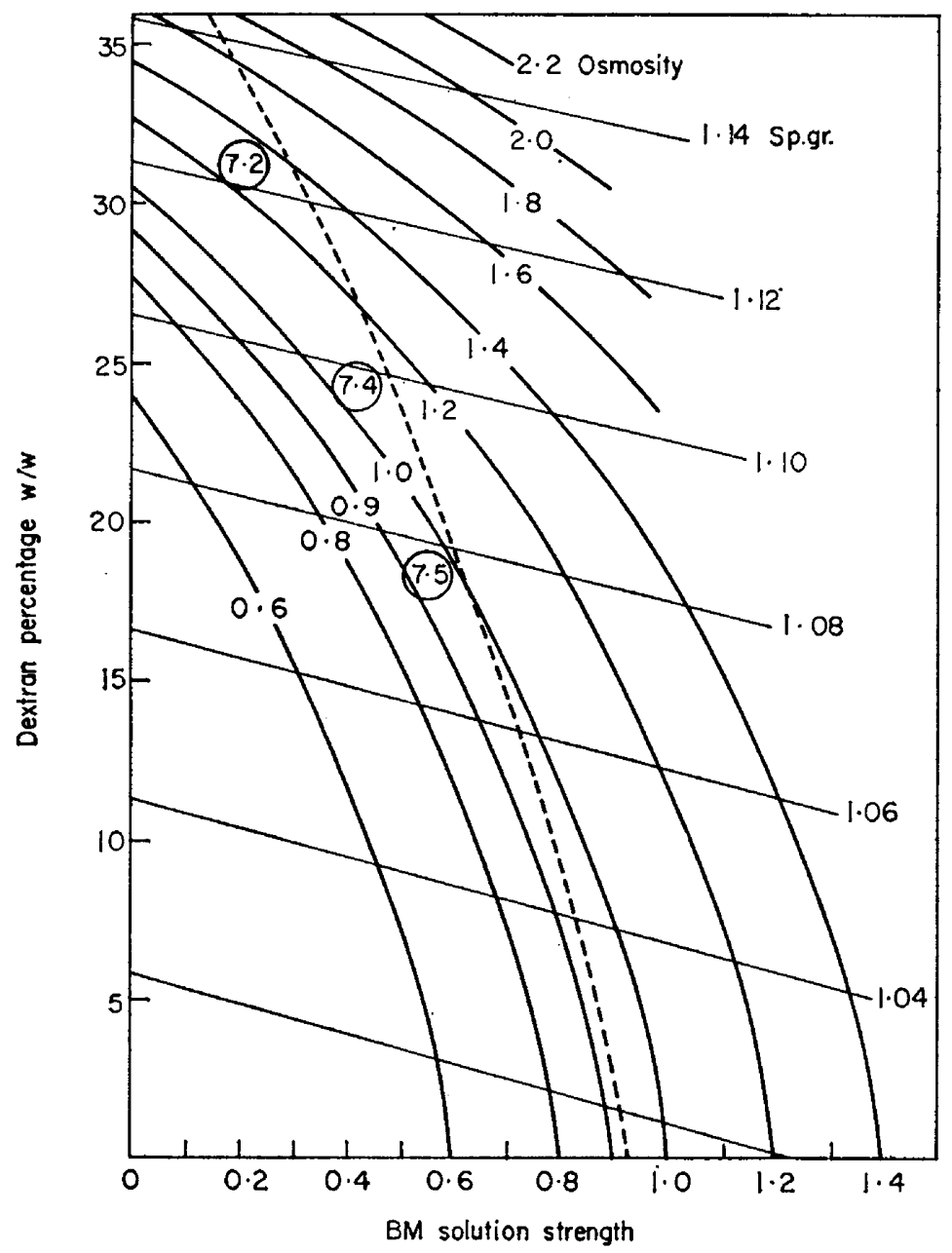

Text-Fig. 1. Properties of a dextran-based density gradient medium. Dextran (ordinate) is dissolved in strengths of BM solution (abscissa). For the resulting mixtures, curves join points of equal osmosity (i.e. $\mathrm{NaCl}$ percentage $\mathrm{w} / \mathrm{v}$ equivalents), near-straight lines join points of equal specific gravity, $20^{\circ} / 20^{\circ} \mathrm{C}$. The dashed line follows roughly the points of maximum spermatozoan motility at a given specific gravity; the circled figures show the approximate pH along that line. Dextran was untreated Ficoll (Pharmacia), Lot To. 5682. BM solution has a strength equal to its osmosity, and $\mathrm{pH} 7 \cdot 6$. 1.0 strength $\mathrm{BM}$ solution is: glucose, $3.362 \mathrm{~g} ; \mathrm{NaCl}, 0.252 \mathrm{~g} ; \mathrm{Na}_{2} \mathrm{HPO}_{4}, 0.252 \mathrm{~g} ; \mathrm{KH}_{2} \mathrm{PO}_{4}, 0.0353 \mathrm{~g}$; distilled water to $100 \mathrm{ml}$.

Four hundred and ninety-two offspring in 82 litters were obtained, not all yielding information for every characteristic under study.

Expected segregation ratios at birth were available from other studies of the same rabbit stocks in this laboratory. $R$ and $r$ constituted an almost 'perfect' Mendelian dominant and recessive. Sex ratio at birth may be taken as $1: 1$ for the 
present purposes, though the actual incidence of males was probably about $48 \%$. At the albino locus (Table 1) the heterozygote and both homozygotes can all be picked out by inspection and divergence from Mendelian expectation in living offspring approaches but does not reach statistical significance at the $0.05 P$ level. But there is a known difficulty in scoring offspring dead at birth, an estimated $36 \%$ of $c^{c h l} c^{c h l}$ being misclassed as $c c^{c h l}$, and $23 \%$ of $c c^{c h l}$ as $c c$. The resulting distorted ratios for dead offspring were estimated as follows from a maximum likelihood calculation designed to integrate the data for dead offspring in the Table. Mating $c c^{c h l} \times c c^{c h l}$ gives (dead) offspring $c^{c h l} c^{c h l}: c c^{c h l}: c c$ in apparent ratio $0 \cdot 16: 0 \cdot 47: 0 \cdot 37$ (or $0 \cdot 63: 0 \cdot 37$ if coloured classes are combined); mating $c c^{c h l} \times c c$ gives (dead) offspring $c c^{c h l}: c c$ in apparent ratio 0.38:0.62. Records of 1059 offspring gave a proportion of 0.845 live at birth.

The results in Table 2 when submitted to various $\chi^{2}$ tests yield no significant evidence of any effect of the buoyant density classes on segregation ratios of sex, rex and albino, and control segregations are consistent with expectation. Hence, there is no evidence of any gross difference in buoyant density between

TABLE 1

SEgREgation OF ALBINo (c) AND LIGHT CHINCHILla $\left(c^{\text {chl }}\right)$ IN RABBIT STOCKS

\begin{tabular}{l|l|ccc}
\hline \multirow{2}{*}{ Mating } & \multicolumn{3}{|c|}{ Status of offspring } & \multicolumn{3}{|c}{ Genotype and numbers of offspring } \\
\cline { 2 - 5 } & & $c^{c h l} c^{c h l}$ & $c c^{c h l}$ & $c c$ \\
\hline$c^{c h l} \times c c^{c h l}$ & Scored live at birth, not weaned & 205 & 455 & 235 \\
$c c^{c h l} \times c c^{c h l}$ & Weaned and scored at 42 days & 146 & 315 & 180 \\
$c^{c h l} \times c c$ & Scored live at birth, not weaned & - & 82 & 82 \\
$c c^{c h l} \times c c^{c h l}$ & Dead births & 27 & 80 & 57 \\
$c c^{c h l} \times c c$ & Dead births & & 6 & 18 \\
\hline
\end{tabular}

$\mathrm{X}$ and Y spermatozoa, nor $R$ and $r$, nor $c$ and $c^{\text {chl }}$. A lack of effect on viability was judged by inspection of the figures; simple $\chi^{2}$ tests do yield a few 'significances' but the tests are suspect because of the familiar tendency towards whole-litter mortality in rabbits.

The birth weight data were analysed after random exclusion of some control litters so that in the final data each litter came from a different doe and each sire gave at least two litters. There were 89 experimental offspring in 17 litters and 122 control offspring in 22 litters. The litter mean of log birth weight was analysed, weighted by litter size. Corrections for various covariance factors chosen in advance of the experiment were incorporated. They included the identity of the five sires; age, weight and parity of doe; litter size and proportion born dead; and gestation period. Correction for gestation period means that birth weights are estimated 'as at constant gestation period' and would therefore be reflections of foetal growth rate. The six observed classes of buoyant density (including controls) named in Table 2 either represent directly, or else are reducible to, combinations of four basic classes: $<1 \cdot 11,1 \cdot 11-1 \cdot 12$, $1 \cdot 12-1 \cdot 13,>1 \cdot 13$. Variation in birth weight attributable collectively to the estimated effects of these basic classes was significant $(0 \cdot 05>P>0 \cdot 025)$. The 
remaining variation between the observed classes was non-significant. The calculation required knowledge of the relative numbers of spermatozoa in the four basic classes in normal semen. This was obtained from the data for silica gradients summarized in Beatty (1964a). After correction for the covariance factors, mean birth weights (antilogs of log means) in the four basic classes were estimated as follows: class $<1 \cdot 11,45 \cdot 7 \mathrm{~g}$; class $1 \cdot 11-1 \cdot 12,51 \cdot 5 \mathrm{~g}$; class $1 \cdot 12-1 \cdot 13,16 \cdot 7 \mathrm{~g}$; class $>1 \cdot 13,57 \cdot 0 \mathrm{~g}$. These estimates are very rough. It had been thought before the experiment that the multifactorial nature of genetic control of birth weight would presumably offer numerous gene segregations among which there would be a better chance of detecting a 'haploid effect' than by study of single segregations, and indeed the results support this expectation. However, because it is difficult to obtain a full statistical understanding in a small experiment of this kind, and because of the modest level of significance attached to a somewhat heterodox result, I regard the apparent effect of buoyant density on birth weight as encouragement for further work rather than as a serious demonstration of a 'haploid effect'. It may be noted that what was technically easier to score or analyse (Mendelian segregations) yielded negative results.

Control of the sex ratio at birth after insemination with immobilized mammalian spermatozoa separated according to their rate of free fall in a viscous medium, i.e. according to some unknown combination of shape, orientation and density, has been claimed by Bhattacharya $(1958,1962)$ and Schilling $(1965,1966)$. This was not confirmed by Andersen \& Rottensten (1962), Bedford \& Bibeau (1967) and Bhattacharya, Bangham, Cro \& Keynes (1966). If one believes that there is an element of success in such experiments, then the present work suggests that differential density of $\mathrm{X}$ and $\mathrm{Y}$ spermatozoa would not be the operative factor. The present work supports the lack of apparent effect on sex ratio in the density-gradient experiments of Benedict, Schumaker \& Davies (1967).

Genetics and biochemistry provide much evidence that the developing or mature gamete is not and cannot be affected by its own genetic content. As against this, the unique and apparently well-established example of a 'haploid effect' in mammalian spermatozoa (Braden, 1958; Yanagisawa, Dunn \& Bennett, 1961) is a continuing stimulus for further research.

I am greatly obliged to Mr Z. Herzenshtein of International Computers Ltd for carrying out multiple regression calculations on birth weight as a demonstration of their ICT 1900 computer; and to Mr Roy Middleton and $\mathrm{Mr}$ J. M. Murison of the Edinburgh Regional Computing Centre for programming and computing the statistical smoothing operations underlying Text-fig. 1. I also acknowledge skilled assistance from Mr W. W. Taylor in the biological aspects of the work.

\section{REFERENCES}

Andersen, H. \& Rottensten, K. (1962) Forsøg med kønskontrol på kaniner. [With English summary]. Ann. Rep., The Royal Veterinary and Agricultural College, Copenhagen; Sterility Research Institute, p. 125. Beatty, R. A. (1964a) Density gradient media for mammalian spermatozoa. Proc. Vth Int. Congr. Anim. Reprod. Artif. Insem. Trento, 1964, 3, 276. 
BEATty, R. A. (1964b) Density gradient media for mammalian spermatozoa. Biol. Bull. mar. biol. Lab. Woods Hole, 127, 354.

BEDFord, J. M. \& BibEAU, A. M. (1967) Failure of sperm sedimentation to influence the sex ratio of rabbits. F. Reprod. Fert. 14, 167.

Benedict, R. G., Schumaker, V. N. \& Davies, R. E. (1967) The buoyant density of bovine and rabbit spermatozoa. F. Reprod. Fert. 13, 237.

Bhattacharya, B. C. (1958) Sex control in mammals. Z. Tierzücht. ZüchtBiol. 72, 250.

Bhattagharya, B. C. (1962) Die verschiedene Sedimentationsgeschwindigheit der X- und Y-spermien und die Frage der willkurlichen Geschlechtsbestimmung. Z. wiss. Zool. 166, 203.

Bhattacharya, B. C., Bangham, A. D., Cro, R. J. \& Keynes, R. D. (1966) An attempt to predetermine the sex of calves by artificial insemination with spermatozoa separated by sedimentation. Nature, Lond. 211, 863.

Braden, A. W. H. (1958) Influence of time of mating on the segregation ratio of alleles at the T locus in the house mouse. Nature, Lond. 181, 786.

Lavon, U., Volcani, R., Amir, D. \& Danon, D. (1966) The specific gravity of bull spermatozoa and seminal plasma. F. Reprod. Fert. 11, 447.

Schilling, E. (1965) Experimentelle Untersuchungen zur Geschlechtsbeeinflussung beim Rind. Naturwissenschaften, 12, 353.

Schilling, E. (1966) Experiments in sedimentation and centrifugation of bull spermatozoa and the sex ratio of born calves. F. Reprod. Fert. 11, 469.

Yanagisawa, K., DunN, L. C. \& Bennetr, D. (1961) On the mechanism of abnormal transmission ratios at $\mathrm{T}$ locus in the house mouse. Genetics, 46, 1635. 